\title{
Aurélie Biard, État, religion et société en Asie centrale post-soviétique. Usages du religieux, pratiques sociales et légitimités politiques au Kirghizstan
}

\section{(2) OpenEdition}

Édition électronique

URL : https://journals.openedition.org/emscat/2719

DOI : $10.4000 /$ emscat.2719

ISSN : 2101-0013

Éditeur

Centre d'Etudes Mongoles \& Sibériennes / École Pratique des Hautes Études

Référence électronique

«Aurélie Biard, État, religion et société en Asie centrale post-soviétique. Usages du religieux, pratiques sociales et légitimités politiques au Kirghizstan », Études mongoles et sibériennes, centrasiatiques et tibétaines [En ligne], 46 | 2015, mis en ligne le 10 septembre 2015, consulté le 13 juillet 2021. URL http://journals.openedition.org/emscat/2719; DOI : https://doi.org/10.4000/emscat.2719

Ce document a été généré automatiquement le 13 juillet 2021.

(C) Tous droits réservés 


\section{Aurélie Biard, État, religion et société en Asie centrale post- soviétique. Usages du religieux, pratiques sociales et légitimités politiques au Kirghizstan}

\section{RÉFÉRENCE}

Thèse de doctorat de Science politique soutenue à l'IEP de Paris, le 29 mai 2015 (556p. + annexe de photos). Membres du jury : S. A. Dudoignon, R. Hamayon, Ch. Jaffrelot, P. Michel (directeur), E. Pace, C. Poujol (rapporteur), S. Serrano (rapporteur)

1 L'effondrement de l'URSS en décembre 1991 a engendré une nouvelle configuration politique, économique et sociale dans l'ensemble de l'espace postsoviétique, dont les effets se font encore sentir plus de vingt ans après. La disparition du régime a offert la possibilité aux populations éponymes de chacune des républiques centrasiatiques (Kazakhstan, Kirghizstan, Ouzbékistan, Tadjikistan, Turkménistan), de réinvestir leur espace religieux, transformé par soixante-dix ans de politique athéiste militante, et de "redéfinir les références symboliques de leur identité, et cela à un niveau qu'elles n'avaient jamais connu $»^{1}$. Par l'établissement d'un État indépendant, ces sociétés sont devenues à la fois les acteurs et les objets de multiples débats concernant leur identité nationale. Ces amples recompositions sociales ont conduit à d'importantes réarticulations du 'croire', celui-ci étant appréhendé comme « entreprise d'allocation de sens $»^{2}$, dans un contexte politique nouveau ${ }^{3}$.

2 Ce travail de thèse explore la difficile reconstruction des dispositifs identitaires suite au flottement généralisé induit par les recompositions dues à la fois à l'effondrement du communisme et à la mondialisation de l'économie et de la culture. Les brutales transformations qu'ont connues les sociétés centrasiatiques cassent en effet les 
références culturelles 'traditionnelles', questionnent les identités, bousculent, dans un univers dynamique marqué par le disruptif, toute stabilité, et nécessitent une reformulation des logiques de légitimité. Cette recherche porte sur le cas du Kirghizstan, république d'Asie centrale turcophone, de tradition nomade et à majorité musulmane (islam hanafi $i^{4}$ ), qui représente un laboratoire à forte valeur heuristique afin d'observer les recompositions identitaires à l'œuvre dans le monde contemporain.

3 L'une des principales stratégies déployées par les acteurs de la scène kirghizstanaise est celle des redistributions réciproques entre religion et politique. Nulle surprise, semble$\mathrm{t}$-il, à ce que le religieux soit convoqué par des acteurs comme vecteur d'ajustement dans un contexte caractérisé par des changements brutaux: la religion est traditionnellement tenue pour un marqueur identitaire particulièrement performant car elle cultive les mythes d'origine et se veut un conservatoire des traditions. Le religieux représente donc, comme le note Patrick Michel, « un registre performant de production d'une stabilité de référence, et dès lors d'énonciation d'une altérité de référence, en situation de flottement généralisé des critères traditionnellement tenus pour pertinents en matière de définition identitaire $»^{5}$.

4 L'on se propose donc, dans ce travail de thèse, d'étudier les réponses apportées par les acteurs de la scène kirghizstanaise aux bouleversements induits par la mise en flottement des critères d'identité traditionnellement pertinents. Leur réponse se fonde principalement sur la ré-articulation du religieux en relation au politique à quatre niveaux différents : celui de la liquidation de l'URSS et, dès lors, de l'empire tsariste dont l'Union soviétique avait hérité ; celui de l'héritage de l'idéologie soviétique et, partant, la liquidation de formes anciennes de légitimité politique et l'émergence de nouvelles légitimités politiques, hybrides et plurielles (souvent encore empreintes des formes anciennes) ; celui de la construction de l'État et de la nation et, enfin, celui de la mondialisation dont le Kirghizstan subit les effets dissolvants.

5 Afin de dégager des éléments d'analyse visant à rendre compte du statut, de la nature et du rôle du religieux sur la scène contemporaine kirghizstanaise, nous explorons la triangulation des acteurs État-religion-société, avec l'objectif de démontrer leur imbrication intime. La thèse centrale de ce travail soutient en effet que la place du religieux dans la société kirghize doit être appréhendée non comme une structure absolue avec des entités hermétiques les unes aux autres; 'religion', 'politique' et 'identité' ; mais comme un processus qui est à la fois dynamique, éminemment hybride et imbriquant de manière intime ces trois répertoires. Il ne s'agit, dès lors, guère d'étudier une structure mais des pratiques discursives et matérielles, individuelles et collectives, qui permettent de comprendre ces interactions qui font que le religieux 'illustre' les changements sociétaux profonds des sociétés post-soviétiques. La religion est en effet un élément clé dans l'instrumentalisation de la nation, dans le quotidien ritualisé de la population locale et dans le rapport au politique. Notre approche remet en cause le postulat selon lequel la religion serait dotée d'une pertinence qui lui appartiendrait en propre alors même que ce qui est décrit n'est jamais tant la 'religion' que les utilisations simultanées et parfois contradictoires dont elle fait l'objet ${ }^{6}$. Le religieux peut en effet aussi bien servir de vecteur à une validation des constructions en cours et/ou de l'ordre établi dans la république kirghize post-soviétique qu'à leur contestation.

6 Ce travail de thèse s'assigne donc pour objet de se saisir des recompositions du religieux comme autant d'indicateurs et de modalités de gestion des évolutions 
affectant la société contemporaine kirghizstanaise, depuis l'effondrement de l'Union soviétique. L'analyse porte non sur l'offre religieuse mais sur l'utilisation qui est faite de ce religieux sur une scène kirghizstanaise traversée par les flux de la mondialisation, de la part d'acteurs, tout à la fois religieux, sociaux et économiques. L'on privilégie dès lors une lecture des emplois et réemplois du religieux sur le terrain politique au Kirghizstan en s'inscrivant dans le cadre d'une sociologie politique des usages de biens symboliques. Pour ce faire, les mutations du religieux, de l'islam en particulier, sont replacées dans leur contexte politique, économique et social. Il s'agit de situer le religieux en objet dans son champ, la société kirghizstanaise de la post-indépendance, et de faire que, dans un va-et-vient constant, le champ révèle l'objet et l'objet le champ $p^{7}$

7 Cette problématique centrale (le religieux indicateur et mode de gestion) ${ }^{8}$ est ici explorée par le biais de trois hypothèses principales correspondant à autant d'usages faits du religieux de la part de différents acteurs.

8 La première hypothèse est celle du religieux utilisé comme tentative de recharge/ réenchantement de l'ordre politique de l'après-indépendance, qui est lui-même à la recherche d'une légitimité nouvelle depuis l'effondrement du communisme. Le religieux est en effet utilisé par l'État kirghiztanais et les élites républicaines pour servir de support à la 'remise en ordre'/consolidation de l'État-nation après le morcellement violent et chaotique des années 1990. L'exaltation et la sacralisation de la nation titulaire sont donc au cœur du projet politique kirghizstanais et la place accordée au religieux y est donc paradoxale, à la fois importante et marginale. Il ne s'agit pas ici de n'importe quel religieux, mais d'un religieux pensé comme irréductible à une supposée 'voie kirghize' ou 'kirghizité'. Or, la place attribuée à l'islam, la religion majoritaire au Kirghizstan comme dans l'ensemble de l'Asie centrale, comme 'religion de la nation' est des plus problématiques. L'islam, dont la portée sociale et une possible politisation remettent en cause la légitimité de l'État, effraie des élites post-soviétiques en quête d'une foi strictement nationale. Préférence a donc été donnée, de manière équivoque, à un religieux pré-islamique, incarné par le tengrisme ou tengrianstvo ("pratiques liées au Ciel »), l'ancienne religion des peuples turciques. La mode du tengrisme permet en effet de réinventer une tradition religieuse qui, contrairement à l'islam, ne peut se poser en concurrente de l'État, et qui incarne la sacralisation ou le ré-enchantement de la nation effectué par les régimes post-soviétiques, et la remise au goût du jour des discours ethnonationalistes (Chapitre I).

9 Cette sacralisation du national comme compensation à la perte de sens du politique soviétique s'accompagne d'une mise sous tutelle de l'islam. La gestion étatique du religieux se caractérise en effet par la promotion officielle d'un islam dit 'traditionnel' et strictement national, censée conjurer le risque présumé que cette religion universaliste ferait peser sur la stabilité et l'unité des nouveaux États. L'islam sert ainsi de vecteur à l'affirmation d'une spécificité nationale : la 'kirghizité' s'incarne en un 'bon' islam qui se reconnaît comme territorialisé et universaliste, séculaire et non transcendant, et adapté aux traditions vernaculaires érigées en valeurs suprêmes par l'État post-soviétique. Ce 'bon islam' promu par les institutions officielles partage avec le tengrisme de nombreux traits, donnant naissance à des luttes de concurrence entre les partisans des deux religions pour le droit à représenter la 'kirghizité'. Mais l'islam dispose également d'une autre fonction, celle de permettre la disqualification de l'ennemi politique, figure abstraite reconstruite à des fins de légitimation du pouvoir, 
et qui s'incarne dans le cas kirghiz par la supposée 'ouzbékité' de tous les courants islamiques ne se reconnaissant pas dans l'islam dit 'officiel'. Courants soufis et néosoufis clandestins ou groupes salafistes, tous se trouvent réunis sous le terme de wahhabî, considéré par les autorités politiques, religieuses, et par la majorité des citoyens kirghizstanais, comme synonyme d'extrémisme' et de terrorisme (Chapitre II).

10 Notre seconde hypothèse est que le religieux permet, au niveau des communautés et des individus, de redistribuer le rapport au sens dans une société désarticulée par les changements brutaux liés à l'effondrement de l'État-providence soviétique. Le recours à l'islam répond en effet à l'effondrement du contrat social soviétique : le religieux étant étroitement intégré au tissu social kirghiz, il en reflète les changements de valeurs et de normes sociales ainsi que les légitimités en compétition. La faillite de l'État pourvoyeur de biens sociaux a contribué à renforcer les métaphores organicistes mettant en parallèle les trajectoires individuelles et familiales avec les trajectoires collectives projetées de la nation. De là découle le rôle prééminent donné à un religieux thérapeutique comme expliquant et 'soignant' les maux aussi bien collectifs qu'individuels. Les représentations du bonheur et du malheur, du corps, de la vie et de la mort sont donc un reflet indirect mais révélateur des interprétations individuelles et collectives du futur politique de la nation : on est malade car la société est malade - du chaos, de la démocratie, de la perte des valeurs - et les rites de passage, en partie les rites funéraires, incarnent le moment suprême où l'ordre politique et l'ordre individuel doivent finalement se réconcilier. L'articulation complexe et problématique entre norme islamique et traditions dites 'ancestrales' ou pratiques thérapeutiques holistiques s'avère donc centrale, accentuée par le rôle majeur de 'pourvoyeur de sens' et/ou de 'réenchantement' que jouent les figures religieuses locales, souvent supposées être dotées de pouvoirs pouvant interférer sur la santé physiologique (physique) et mentale (Chapitre III).

11 Notre troisième et dernière hypothèse est que le religieux est également utilisé pour établir un rapport renouvelé au politique et vise, partant, une refondation de l'ordre politique. En Asie centrale comme ailleurs, l'islamisme politique des décennies 1980-1990 cède progressivement le pas devant un néo-fondamentalisme conservateur qui veut réformer la société et les mœurs et faire revivre l'islam primitif. Ce qui est remis en cause, c'est non seulement le culturel mais également le politique. L'on assiste ainsi, chez certains mouvements comme le Hizb al-Tahrîr, d'origine proche-orientale, partout interdit en Asie centrale, à une réactivation, via la rhétorique locale de l'idéal du califat, d'une utopie religieuse contestataire de l'ordre politique et économique. De son côté, le mouvement revivaliste et prosélyte, né dans le sous-continent indien, du Tablighi Jama'at, lequel jouit au Kirghizstan d'une large marge de manœuvre en raison de son 'apolitisme proverbial', a certes « mis entre parenthèses les questions de prise de pouvoir ", mais sans pour autant "écarter le but ultime de voir les musulmans partout au pouvoir $»^{10}$. Le Tabligh se concentre en effet sur la formation d'un noyau de fidèles 'authentiques', de born again, et apparaît dès lors comme un mouvement supranational qui vise à réactiver la solidarité politique des musulmans quelle que soit leur nationalitée ${ }^{11}$, un défi sans précédent pour l'État kirghiz et sa vision territorialisée et sacralisée de la nation (Chapitre IV). 


\section{BIBLIOGRAPHIE}

Gaborieau, M.

1997 Renouveau de l'islam ou stratégie politique occulte ? La Tablîghî Jamâ'at dans le souscontinent indien et dans le monde, in C. Clémentin-Ojha (dir.), Renouveaux religieux en Asie (Paris, École française d'Extrême-Orient), pp. 211-229.

Hamayon, R.

1996 Chamanisme, bouddhisme, héroïsme épique : quel support d'identité pour les Bouriates post-soviétiques, Études mongoles et sibériennes, 27, pp. 327-358.

Kepel, G.

2012, Le Prophète et Pharaon. Les mouvements islamistes dans l'Égypte contemporaine (Paris, Gallimard).

Michel, P.

1993, Pour une sociologie des itinéraires de sens : une lecture politique du rapport entre croire et institution - hommage à Michel de Certeau, Archives de Sciences Sociales des Religions, 82,

pp. 223-238.

1999, Religion, nation et pluralisme - Une réflexion fin de siècle, Critique internationale, 3 , pp. 79-97.

2006, Espace ouvert, identités plurielles : les recompositions contemporaines du croire, Social Compass, 53, 2, p. 227-241.

Michel, P., \& J. García-Ruiz

2012 Et Dieu sous-traita le Salut au marché. De l'action des mouvements évangéliques en Amérique latine (Paris, Armand Colin).

Muminov, A.

1999 Traditional And Modern Religious-Theological Schools in Central Asia, in L. Jonson \& M. Esenov (dir.), Political Islam and Conflicts in Russia and Central Asia (Stockholm, The Swedish Institute of International Affairs), pp. 101-111.

\section{NOTES}

1. Hamayon 1996, p. 327.

2. Il s'agit, comme le précise Patrick Michel, d'une approche par le 'croire' et non par la 'croyance', ou a fortiori par la 'religion'. «Chacun de ces deux derniers termes est en réalité tellement marqué par la référence à la stabilité fictive qu'il apparaît impropre à décrire une réalité organisée par le mouvement. Le croire est le dispositif, nécessairement dynamique et donc évolutif, par lequel du sens est recherché et affecté » $(1999$, p. 82).

3. Michel 1993, p. 225.

4. Muminov 1999, pp. 101-111.

5. Michel 2006, p. 229.

6. García-Ruiz \& Michel 2012.

7. Kepel 2012, p. 285.

8. Sur cette problématique, voir Michel 2006, pp. 227-241.

9. Le Kirghizstan est la seule république d'Asie centrale à ne pas avoir banni le Tablighi Jama'at, ce mouvement transnational est très actif dans le pays.

10. Gaborieau 1997, p. 220. 
11. Gaborieau Op. cit. p. 223. 